\title{
Investigation of sumatriptan and ketorolac trometamol in the human experimental model of headache
}

\author{
Hashmat Ghanizada', Mohammad Al-Mahdi Al-Karagholi', Nanna Arngrim', Mette Mørch-Rasmussen',
} Matias Metcalf-Clausen ${ }^{1}$, Henrik Bo Wiberg Larsson ${ }^{2}$, Faisal Mohammad Amin ${ }^{1}$ and Messoud Ashina ${ }^{1 *}$

\begin{abstract}
Background: Pituitary adenylate cyclase-activating polypeptide-38 (PACAP38) induces headache in healthy volunteers but the precise mechanisms by which PACAP38 leads to headache are unclear. We investigated the headache preventive effect of sumatriptan and ketorolac on PACAP38-induced headache in healthy volunteers. In addition, we explored contribution of vascular mechanisms to PACAP38-induced headache using high resolution magnetic resonance angiography.
\end{abstract}

Methods: Thirty-four healthy volunteers were divided in two groups (A and B) and received infusion of PACAP38 (10 picomol/kg/min) over $20 \mathrm{~min}$. Group A was pretreated with intravenous sumatriptan (4 mg) or ketorolac $(30 \mathrm{mg})$ 20 min before infusion of PACAP38. Group B received infusion of sumatriptan or ketorolac as post-treatment $90 \mathrm{~min}$ after infusion of PACAP38. In both experiments, we used a randomized, double-blind, cross-over design. We recorded headache characteristics and circumference of extra-intracerebral arteries.

Results: We found no difference in AUC (0-6 h) of PACAP38-induced headache in group A, pretreated with sumatriptan or ketorolac $(p=0.297)$. There was no difference between sumatriptan and ketorolac in PACAP38induced circumference change (AUC Baseline-110 min) of MMA $(p=0.227)$, STA $(p=0.795)$ and MCA $(p=0.356)$. In group B, post-treatment with ketorolac reduced PACAP38-headache compared to sumatriptan $(p<0.001)$. Posttreatment with sumatriptan significantly reduced the circumference of STA $(p=0.039)$ and MMA $(p=0.015)$ but not of MCA ( $p=0.981)$ compared to ketorolac. In an explorative analysis, we found that pre-treatment with sumatriptan reduced PACAP38-induced headache compared to no treatment ( $A \cup \mathrm{C}_{0-90 \mathrm{~min}}$ ).

Conclusions: Post-treatment with ketorolac was more effective in attenuating PACAP38-induced headache compared to sumatriptan. Ketorolac exerted its effect without affecting PACAP38-induced arterial dilation, whereas sumatriptan post-treatment attenuated PACAP38-induced dilation of MMA and STA. Pre-treatment with sumatriptan attenuated PACAP38-induced headache without affecting PACAP38-induced arterial dilation. Our findings suggest that ketorolac and sumatriptan attenuated PACAP38-induced headache in healthy volunteers without vascular effects.

Trial registration: Clinicaltrials.gov (NCT03585894). Registered 13 July 2018,

Keywords: Headache, PACAP38, Mast cell degranulation, Plasma protein extravasation, Arterial dilation, Neuroinflammation, Pain, NSAIDs, MRA

\footnotetext{
* Correspondence: ashina@dadlnet.dk

1Danish Headache Center and Department of Neurology, Rigshospitalet

Glostrup, Faculty of Health and Medical Sciences, University of Copenhagen,

Valdemar Hansens Vej 5, DK-2600 Glostrup, Denmark

Full list of author information is available at the end of the article
}

(c) The Author(s). 2020 Open Access This article is distributed under the terms of the Creative Commons Attribution 4.0 International License (http://creativecommons.org/licenses/by/4.0/), which permits unrestricted use, distribution, and reproduction in any medium, provided you give appropriate credit to the original author(s) and the source, provide a link to the Creative Commons license, and indicate if changes were made. 


\section{Background}

Pituitary adenylate cyclase-activating polypeptide 38 (PACAP38) is a pleiotropic signaling neuropeptide [33, $34]$ that induces headache in healthy volunteers and migraine attacks in migraine patients [4, 42]. PACAP38 is located in both sensory and parasympathetic perivascular nerve fibers [35,52] and its infusion causes prolonged extracerebral dilation $[5,7,13,20,49]$ and dural mast cell degranulation [12]. Recent data showed that PACAP38 activated mast cell specific receptor Masrelated G-protein-coupled receptors-b2 (Mrgprb2) [22, 38 ] which mediates neurogenic inflammation and pain [22]. Activation of mast cells leads to recruitment of dural immune cells involving neutrophils, monocytes and macrophages $[25,40,47]$. Dural neurogenic inflammation and mast cell mediated activation of the trigeminal pain pathway have been suggested to play a key role in migraine pathogenesis $[31,36]$. The precise mechanisms by which PACAP38 leads to headache and migraine are unclear.

The anti-migraine-specific drug sumatriptan, a $5-\mathrm{HT}_{1 \mathrm{~B}}$ /1D agonist [18], is a vasoconstrictor [6, 27] with antiinflammatory properties [16] that potently blocks neurogenic plasma extravasation from dural blood vessels [17]. Ketorolac is cyclooxygenase (COX-1 and COX-2) inhibitor non-steroidal anti-inflammatory drug [41, 44, 48] that reduces mast cell degranulation [50] and blocks dural macrophage activation [37]. The neurovascular effects of ketorolac have not been studied in humans. Sumatriptan and ketorolac are used as abortive medication for migraine treatment but the site and mode of action of these drugs are not fully clarified.

In the present study, we used PACAP38 as a biomarker of headache with inflammatory and vascular components. To further elucidate the mechanisms underlying the action of sumatriptan and ketorolac, we investigated the effect of both drugs on PACAP38-induced headache in healthy volunteers. We hypothesized that both sumatrip$\tan$ and ketorolac would attenuate PACAP38-induced headache but only sumatriptan infusion would abolish PACAP38-induced arterial dilation. To test this hypothesis we conducted a randomized, double-blind, crossover study and used magnetic resonance angiography (MRA) to record vascular responses.

\section{Methods}

\section{Participants}

We recruited thirty-four healthy volunteers. All participants were pre-screened over telephone and all potential study candidates were invited to the hospital for thorough screening. The eligibility criteria for inclusion in the study were as follows; adults $\geq 18$ to $\leq 50$ years of age of both sexes with body weight of 50 to $100 \mathrm{~kg}$. Exclusion criteria included: daily intake of any medication except contraceptives, magnetic resonance imaging contraindications, serious somatic disease (including any pain condition), history of migraine or any other type of headache expect episodic tension-type headache less than once a month. All participants provided detailed oral and written information about the study and written informed consent was obtained in accordance with the Helsinki declarations. The study was approved by the Ethics Committee of the Capital Region of Denmark (H18008313) and registered at Clinicaltrials.gov (ID: NCT03585894).

\section{Experimental design}

We divided participants into two groups: group $\mathrm{A}$ and group B. In group A, participants were randomly assigned to intravenous infusion of sumatriptan $4 \mathrm{mg}$ (GlaxoSmithKline Pharma A/S, Denmark) or ketorolac trometamol $30 \mathrm{mg}$ (Atnahs Pharma, UK Limited) over $10 \mathrm{~min}$. At $20 \mathrm{~min}$ after start of infusion of sumatriptan and ketorolac participants received infusion of PACAP38 (10 picomole $/ \mathrm{kg} / \mathrm{min}$ ) [42] over $20 \mathrm{~min}$ (Fig. 1 a). In group B, participants first received PACAP38 infusion over $20 \mathrm{~min}$ and at $90 \mathrm{~min}$ after start of infusion randomly assigned to receive infusion of sumatriptan or ketorolac (Fig. 1 b). In each group, experiments were conducted on two separate days with a washout period of one week. The PACAP38 (Bachem) solution was prepared for the study by the Capital Region Hospital Pharmacy.

All participants were non-fasting and arrived at the hospital $24 \mathrm{~h}$ headache free and were instructed not to consume coffee, tea, cola, caffeine, alcohol, smoking and avoid exhausting physical activities for $12 \mathrm{~h}$ prior to the study day. On arrival, female participants were tested for pregnancy on both days and a venous catheter (Venflon, Becton Dickinson Infusion Therapy $\mathrm{AB}$, Sweden) was inserted into the antecubital vein for infusion. After 15 min of rest in the supine position, headache intensity and characteristics, vital signs, mean arterial blood pressure (MAP), and heart rate (HR) were recorded at baseline and at fixed intervals every $10 \mathrm{~min}(0-120 \mathrm{~min})$. All participants underwent three MRI sessions: baseline, 20 min and $110 \mathrm{~min}$ (Fig. 1). All experiments were conducted in the afternoon due to limited availability of the MRI scanner. We were therefore only able to record headache data from all participants from 0 to $6 \mathrm{~h}$ since most participants went to sleep thereafter.

\section{Headache}

We used a validated headache questionnaire to record headache intensity using numerical rating scale from 0 to 10 (0: no headache; 1 : a very mild headache (including a feeling of pressing or throbbing pre-pain); 10: worst imaginable headache) and associated symptoms. Headache characteristics (localization, quality, aggravation by 
A)

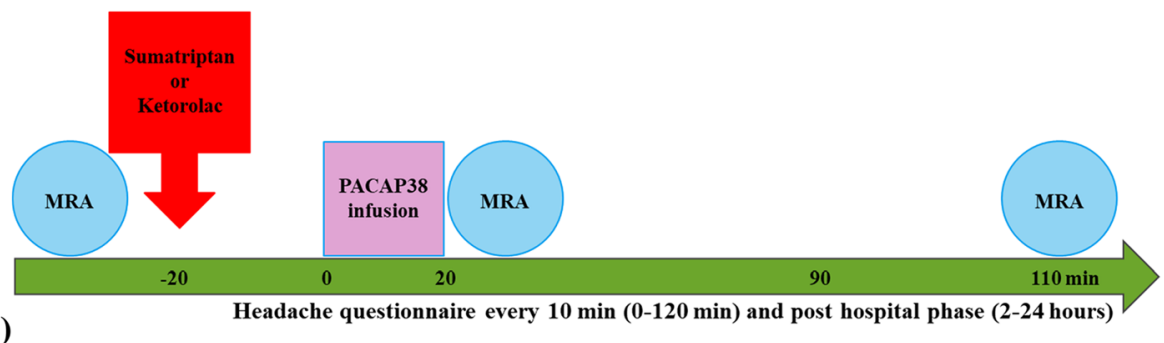

B)

)
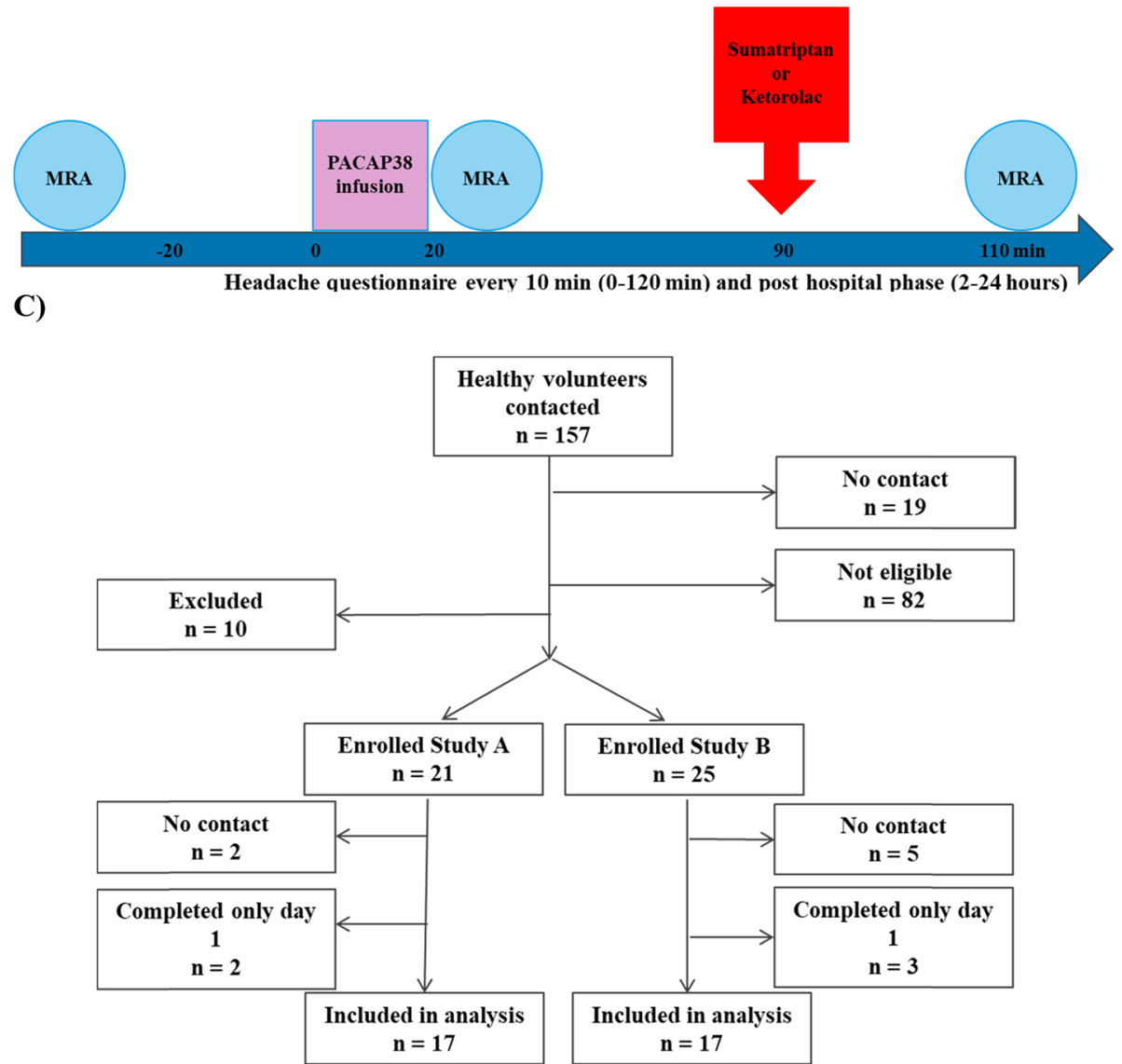

Fig. 1 a Pre-treatment study design. b Post-treatment study design. c Recruitment flow chart. All participants took part in each group in two experimental days separated by one week

physical activity, associated symptoms (nausea, photoand phonophobia)) and prodromes (unusual fatigue, yawning, thirst, craving, mood swings, flushing and difficulty concentrating) were also recorded.

\section{Data acquisition and image analysis}

All scans were performed on a Philips $3 \mathrm{~T}$ Achieva MRI scanner (Philips Medical Systems, Best, The Netherlands) using a 32-channel phase array head coil. A 3D time-of- flight MRA of extra-intracerebral arteries was acquired as described in previous studies [5, 6]. All acquired MRA data were saved in DICOM format and transferred to a separate workstation and analyzed by LAVA-MRA vessel wall analysis software program. LAVA-MRA was previously used in several studies $[4,8]$ and the method demonstrated a low $<5 \%$ inter- and intra-observer variation [3]. The software automatically detects the vessel contours and calculate the circumference every $0.2 \mathrm{~mm}$ perpendicular to the 
center line. For each vessel an average of 26 slides $(5 \mathrm{~mm})$ was obtained and the measurement was repeated for all participant at the same vessel segment.

Two bilateral branches of superficial temporal artery (STA), middle meningeal artery (MMA) and middle cerebral artery (MCA) were analyzed by an investigator who was blinded to the experimental day and scan session.

\section{Statistical analysis}

All absolute values are presented as mean with 95\% confidence interval (CI). We calculated the study sample size based on headache and vascular responses to PACAP38 taking the previous findings into consideration [5]. The risk of type 1 error at 5\% and a defined power at $80 \%$ and type 2 error was fixed at $20 \%$. We calculated that thirty-four participants would be adequate for a crossover study design.

The primary endpoints of the study were a difference in the area under the curve (AUC) for headache (0-6h) and arterial circumference change of STA, MMA and MCA from baseline to 110 min between treatment with sumatriptan and ketorolac. The secondary endpoints were difference in AUC for HR (0-120 min), MAP (0$120 \mathrm{~min})$ and associate symptoms $(0-24 \mathrm{~h})$. Baseline values were subtracted before calculating AUC to reduce within-participant variation between sessions. The nonparametric Wilcoxon signed rank test was used to calculate the side to side difference in arterial circumference. We used Mann-Whitney test to compare the differences between the groups.

All statistical analyses were performed with SPSS version 23.0 (Chicago, IL, USA). We did not adjust for multiple comparisons, as our primary endpoints, hypotheses and statistical tests were all predefined and clearly stated in the study protocol. Five percent $(p<0.05)$ was accepted as the level of significance.

\section{Results}

Thirty-four healthy volunteers (18 females and 16 males, mean age 25 years, (range $19-39$ years) and mean weight of $70 \mathrm{~kg}$, (range $54-88 \mathrm{~kg}$ )) completed the study.
PACAP38 infusion induced headache in sixteen out of seventeen (94\%) subjects pre-treated (10 females and 7 males) with ketorolac and thirteen out of seventeen (77\%) pre-treated with sumatriptan. In post-treatment group (8 females and 9 males), PACAP38 infusion induced headache in sixteen out of seventeen (94\%) treated with ketorolac and seventeen out of seventeen $(100 \%)$ treated with sumatriptan. (Table 1).

We found no difference in arterial circumferences and vital variables at baseline between sumatriptan and ketorolac day in both study groups (A and B). There was no carry-over or period effect for baseline values between the study days. We found no difference between the right and left sided arteries $(p>0.05)$ and therefore, an average of both arteries was used.

\section{Effect of pre-treatment of sumatriptan or ketorolac: group A}

We found no difference in AUC $(0-6 \mathrm{~h})$ for PACAP38induced headache between sumatriptan and ketorolac $(p=0.297)$ (Fig. 2, Table 1). There was no difference in PACAP38-induced circumference change $\left(A U C_{\text {Base- }}\right.$ line-110 min $)$ of MMA $(p=0.227)$, STA $(p=0.795)$ and MCA $(p=0.356)$ after sumatriptan compared to ketorolac (Fig. 3).

\section{Effect of post-treatment of sumatriptan or ketorolac: group B}

We found that ketorolac reduced the AUC $(1.5-6 \mathrm{~h})$ for PACAP38-induced headache compared to sumatriptan $(p<0.001)$ (Fig. 2, Table 1). Sumatriptan significantly reduced the circumference of STA $(p=0.039)$ and MMA $(p=0.015)$ compared to ketorolac. No difference was found in circumference change of MCA $(p=0.981)$ (Fig. $3)$.

\section{Explorative analyses on headache data}

To explore whether both drugs prevent PACAP38induced headache we conducted an explorative analysis on headache data between group A and B from 0 to 90 min: pretreatment with sumatriptan or ketorolac

Table 1 Headache incidence and characteristics after PACAP38 infusion and treatment with ketorolac and sumatriptan from 0 to 2 and $2-6 \mathrm{~h}$. Headache inductions rate: number of participants who developed headache; Median peak headache: peak headache intensity recorded on a numerical rating scale from 0 to 10

\begin{tabular}{|c|c|c|c|c|c|c|}
\hline PACAP38 infusion/treatment & $\begin{array}{l}0-2 \mathrm{~h} \\
\text { Headache } \\
\text { induction rate }\end{array}$ & $\begin{array}{l}2-6 \mathrm{~h} \\
\text { Headache } \\
\text { induction rate }\end{array}$ & $\begin{array}{l}0-2 \mathrm{~h} \\
\text { Median Peak } \\
\text { headache (range) }\end{array}$ & $\begin{array}{l}2-6 \mathrm{~h} \\
\text { Median Peak } \\
\text { headache (range) }\end{array}$ & $\begin{array}{l}0-2 \mathrm{~h} \\
\text { Median Duration } \\
\text { of headache (hours) }\end{array}$ & $\begin{array}{l}2-6 \mathrm{~h} \\
\text { Median Duration } \\
\text { of headache (hours) }\end{array}$ \\
\hline Pre-treatment Ketorolac & 16 of 17 & 13 of 17 & $1(0-5)$ & $0(0-3)$ & 1 & 2 \\
\hline Pre-treatment Sumatriptan & 13 of 17 & 8 of 17 & $1(0-4)$ & $0(0-5)$ & 0.17 & 1 \\
\hline Post-treatment Ketorolac & 16 of 17 & 12 of 17 & $1(0-5)$ & $1(0-2)$ & 1.17 & 4 \\
\hline Post-treatment Sumatriptan & 17 of 17 & 12 of 17 & $2(0-7)$ & $1(0-4)$ & 1.67 & 2 \\
\hline
\end{tabular}



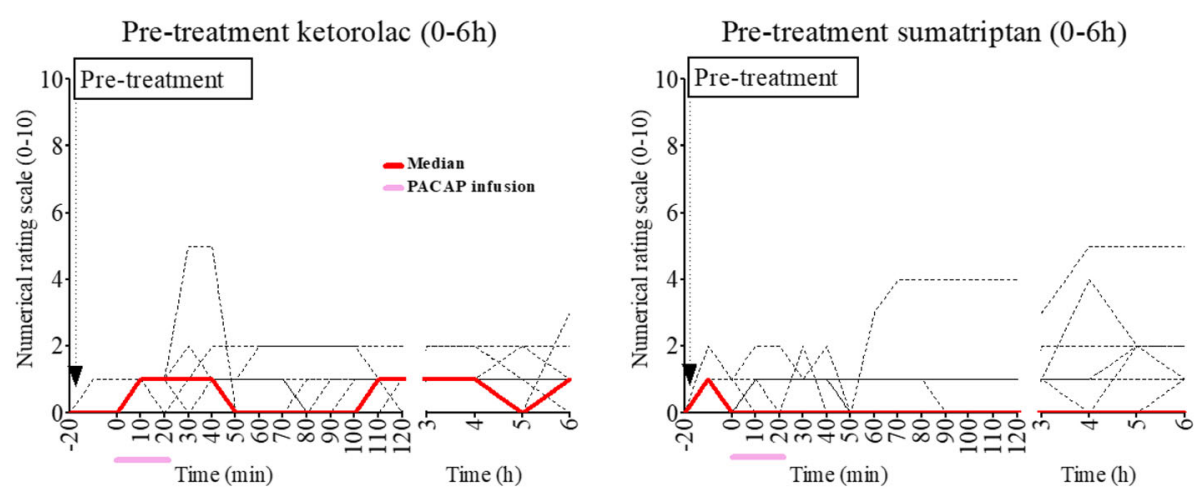

Post-treatment ketorolac $(0-6 \mathrm{~h})$

Post-treatment sumatriptan $(0-6 \mathrm{~h})$
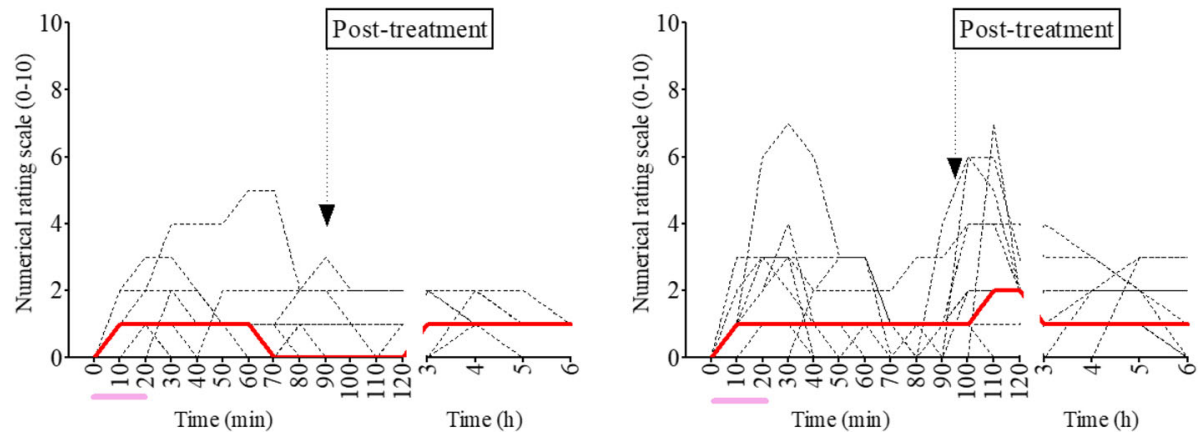

Fig. 2 Headache intensity of individual volunteers (black lines) and the median headache intensity (red line) after PACAP38 and pre-treatment with ketorolac /sumatriptan $(n=17)$ and post-treatment with ketorolac /sumatriptan $(n=17)$

followed by PACAP38 infusion compared to group B where participants received only PACAP38 infusion up to 90 mins. The AUC (0-90 min) for headache score was significantly larger after PACAP38 infusion compared to PACAP38-induced headache pre-treated with sumatrip$\tan (p=0.005)$. A trend of attenuation of PACAP38induced headache was recorded in those who were pretreated with ketorolac compared to only PACAP38 infusion $(p=0.076)$ (Fig. 2).

\section{Vital variables and adverse events}

In pre-treatment group $\mathrm{A}$, we found no difference in AUC (Baseline-120 min) for MAP changes between ketorolac and sumatriptan $(p=0.523)$. In post-treatment group $\mathrm{B}$, the AUC (Baseline-120 min) for MAP was significantly larger after sumatriptan compared to ketorolac $(p=0.028)$. We found no change in AUC (Baseline-120 min) for heart rate in pre-treatment group between sumatriptan and ketorolac $(p=0.492)$ and post-treatment group between sumatriptan and ketorolac $(p=0.356)$ (Fig. 4).

There was no difference in incidence of nausea, difficulty concentrating, neck stiffness, fatigue, thirst and facial puffiness (Table 2).

\section{Discussion}

The main findings of the present study were: 1) Participants who were pre-treated with sumatriptan or ketorolac reported no difference in PACAP38-induced headache; 2) Post-treatment with ketorolac was more effective in attenuating PACAP38-induced headache compared to sumatriptan. Interestingly, the ketorolac exerted its effect without affecting PACAP38-induced arterial dilation, whereas sumatriptan post-treatment attenuated PACAP38-induced dilation of MMA and STA. Explorative analysis showed that pre-treatment with sumatriptan attenuated PACAP38induced headache without affecting PACAP38-induced arterial dilation. In the current study both drugs given as preor post-treatment had no effect on PACAP38-induced autonomic symptoms such as palpitation, facial flushing and heat sensation. Most participants reported facial flushing and puffiness persisting over $24 \mathrm{~h}$ post PACAP38 infusion. PACAP is found in human skin [53] and PACAP infusion causes intense facial flushing [21].

\section{Experimentally induced headache and anti-migraine medication}

Similar to previous studies [5, 42], we found that PACAP38 infusion caused prolonged mild to moderate 

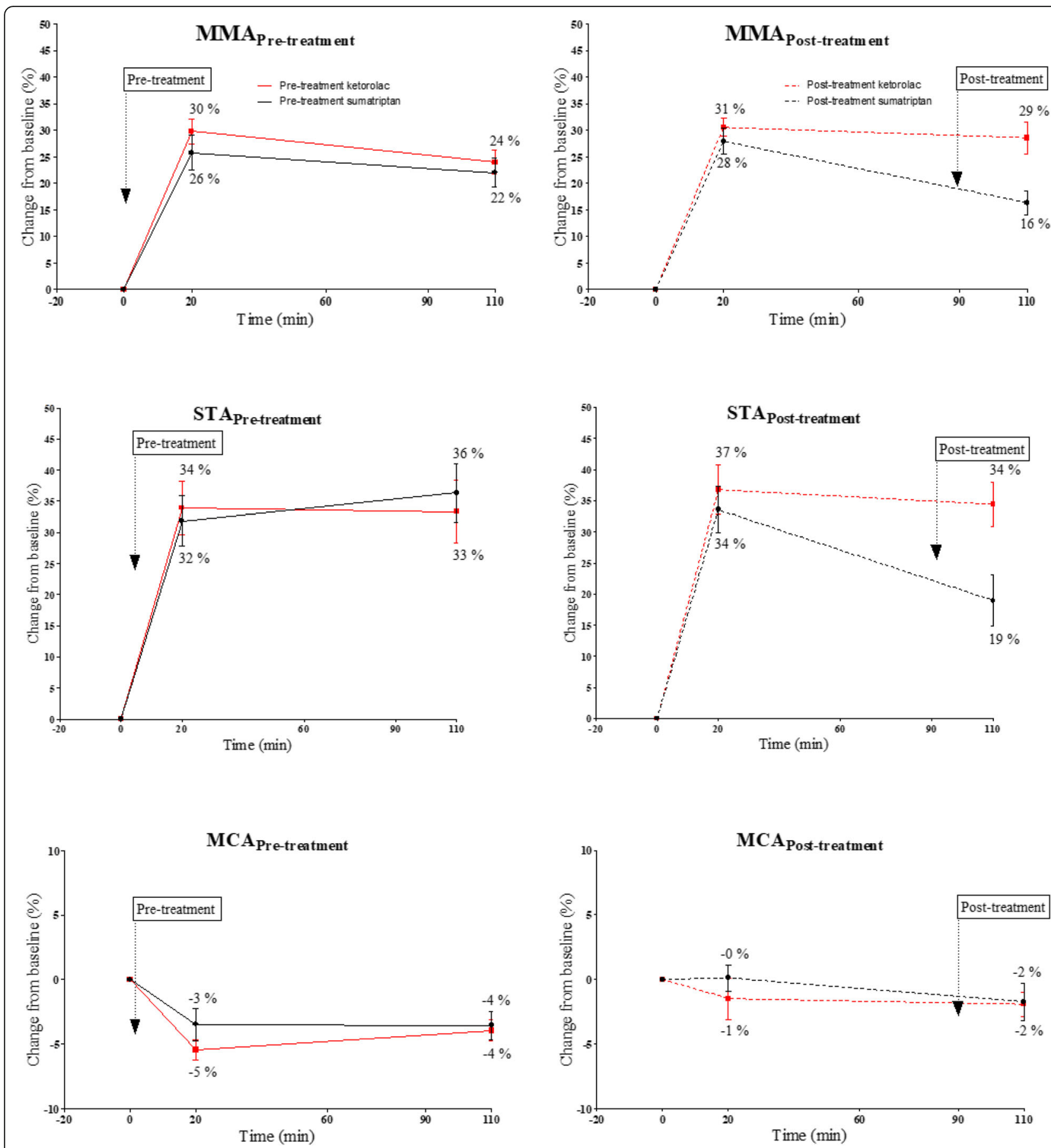

Fig. 3 Effect of pre-treatment (solid line) and post-treatment (dotted line) on extra-intracerebral arteries dilated with PACAP38. Middle meningeal artery (MMA), superficial temporal artery (STA) and middle cerebral artery (MCA)

headache in $90 \%$ of healthy volunteers. The headache preventive effect of sumatriptan when given before PACAP38 infusion is consistent with previous studies demonstrating that pre-treatment with sumatriptan reduced glycerol trinitrate (GTN) [28] and cilostazol [19] induced headache in healthy volunteers. GTN is a prodrug for nitric oxide (NO) and cilostazol is a phosphodiesterase 3 inhibitor which works downstream in the cascade of events associated to headache $[19,28]$. It has been suggested that sumatriptan prevents headache in these models by inhibiting accumulation of cyclic guanosine monophosphate (cGMP) and cyclic adenosine monophosphate (cAMP). Interestingly, posttreatment with triptans failed to antagonize GTN and 

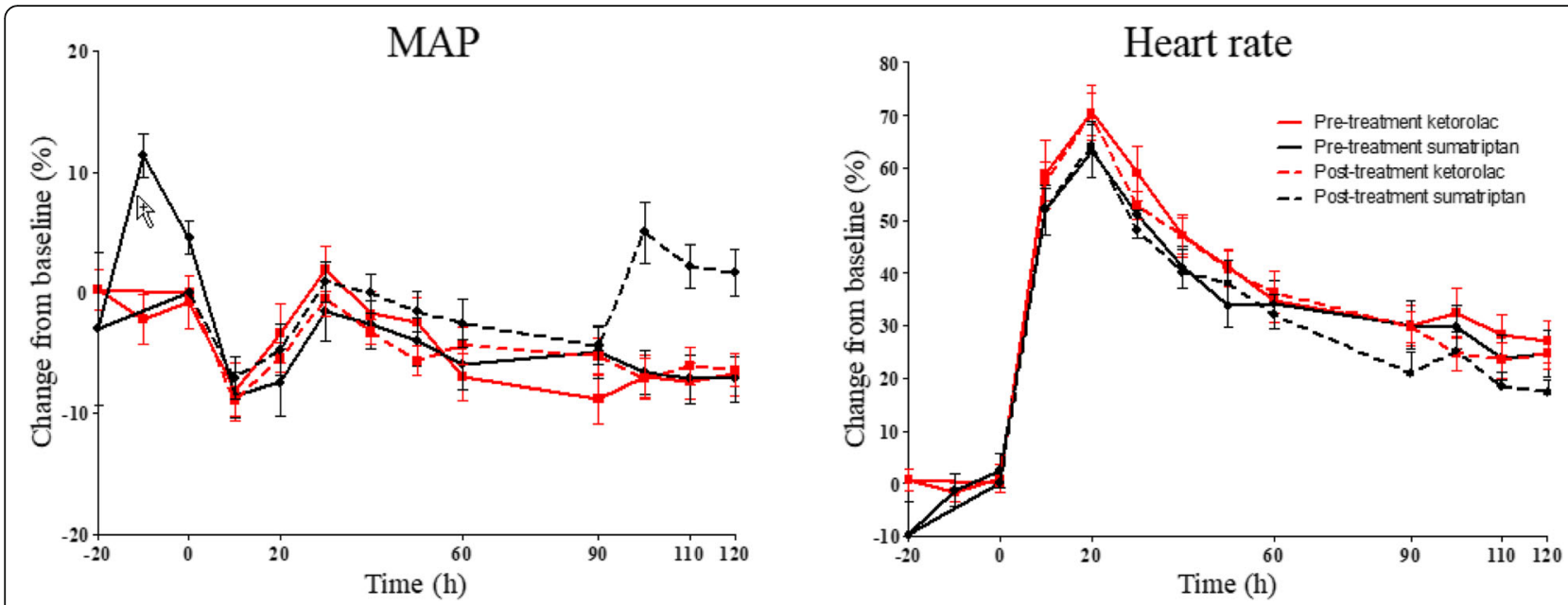

Fig. 4 Percentage change from baseline for mean arterial pressure (MAP) and heart rate (HR) after PACAP38 and pre-treatment and posttreatment with ketorolac and sumatriptan

cilostazol induced headache in healthy volunteers [23, 46]. In line with this, we also show that post-treatment with intravenous administration of sumatriptan failed to prevent PACAP38-induced headache in healthy volunteers. The failure of sumatriptan to abort PACAP38induced headache might be due to establishment of central sensitization and disruption of presynaptic $5-\mathrm{HT}_{1 \mathrm{~B}}$ /1D receptors in the dorsal horn [32].

The lower AUC for headache score (2-6h) after ketorolac compared to sumatriptan post-treatment suggests that ketorolac was more effective than sumatriptan when administered as post-treatment during the established PACAP38-induced headache phase in healthy volunteers. Our findings are consistent with previous studies demonstrating that ketorolac infusion is able to terminate established peripheral and central sensitization [29]. However, our results should be interpreted with caution because sumatriptan infusion caused immediate but short exacerbation of PACAP38-induced headache. The sumatriptan-induced headache was previously observed after infusion of calcitonin gene-related peptide
(CGRP), levcromakalim, isosorbide-5-mononitrate (NO donor) and cilostazol in healthy volunteers [2, 9, 23, 24]. It is also a well-known side effect of sumatriptan treatment in migraine patients $[15,39]$. This may explain the difference between the two drugs administered as posttreatment. However, the similar incidence of headache (Table 1) and unchanged median headache score (Fig. 2) for both drugs in post-hospital phase indicates that both drugs are not effective in aborting PACAP38-induced headache during this phase.

\section{Cranial artery dilation and anti-migraine medication}

Similar to previous studies $[4,5]$, we found that PACAP38 infusion caused sustained dilation of extracerebral arteries but not intracerebral arteries. Though earlier studies have shown that sumatriptan constricts extracerebral arteries $[5,6]$, sumatriptan pre-treatment was unable to counteract PACAP38-induced dilation of MMA and STA. This result shows that PACAP38 bypass the vasoconstrictive effect of sumatriptan in these arteries in healthy volunteers. The headache preventive effect

Table 2 Adverse events after PACAP38 infusion and treatment with ketorolac and sumatriptan

\begin{tabular}{lllll}
\hline Variables & Pre-treatment (Ketorolac) & Pre-treatment (Sumatriptan) & Post-treatment (Ketorolac) & Post-treatment (Sumatriptan) \\
\hline Palpitation & $100 \%$ & $94.1 \%$ & $100 \%$ & $100 \%$ \\
Flushing & $100 \%$ & $100 \%$ & $100 \%$ & $100 \%$ \\
Heat sensation & $100 \%$ & $100 \%$ & $100 \%$ & $100 \%$ \\
Nausea & $0 \%$ & $0 \%$ & $0 \%$ & $0 \%$ \\
Neck stiffness & $11.8 \%$ & $11.7 \%$ & $17.7 \%$ & $17.7 \%$ \\
Difficulty concentrating & $5.9 \%$ & $5.9 \%$ & $23.5 \%$ & $17.8 \%$ \\
Fatigue & $23.5 \%$ & $17.7 \%$ & $23.5 \%$ & $23.5 \%$ \\
Thirst & $17.7 \%$ & $11.8 \%$ & $11.8 \%$ & $23.5 \%$ \\
Facial puffiness & $41.2 \%$ & $35.3 \%$ & $41.2 \%$ & $17.7 \%$ \\
\hline
\end{tabular}


of sumatriptan given before PACAP38 infusion without affecting the vasodilatory response to PACAP38 suggest that the headache inducing effect of PACAP38 is independent of its vasoactive property in healthy volunteers.

In the present study, we found that post-treatment with sumatriptan but not ketorolac reduced MMA and STA circumference, but the dilation of MMA and STA did not return to baseline during the observation period. In humans, sumatriptan constricts normal and predilated extracerebral arteries $[5,6,10]$. In healthy volunteers, subcutaneous injection of sumatriptan totally abolished a modest CGRP induced dilation of MMA [11]. Taken together, it seems that PACAP38-induced vasodilation mechanistically differs from CGRP and its prolonged dilation of extracerebral arteries might be caused via activation of dural mast cells [12]. Identification of a putative new PACAP-receptor on mast cells provides important insight on PACAP38-induced prolonged dilation of extracerebral arteries [38]. Dural mast cells are found in close proximity to meningeal nociceptors, whereupon activation releases vasoactive neuropeptides which mediates activation of pain pathways [22].

To the best of our knowledge, no study investigated possible vasoactive properties of ketorolac using advanced MRA method. Our data demonstrated that ketorolac had no vascular effect on extra-intracerebral arteries and neither pre nor post-treatment alters the vascular effects of PACAP38. It is possible that proinflammatory prostanoids are released by PACAP38 induced mast cell degranulation [12, 30, 45] and the headache attenuating effect of ketorolac treatment might be via inhibition of pro-inflammatory prostanoids activated by PACAP38 infusion.

\section{Mechanisms behind the anti-nociceptive effect of sumatriptan and ketorolac}

It has been reported that PACAP38 induces sensitization of trigeminal neurons via activation of neuronal $\mathrm{PAC}_{1}$ receptor [1]. In animals, pre-treatment with sumatriptan effectively blocked the development of all aspects of central sensitization by blocking the peripheral signal transmission from the meningeal nociceptors [14]. Moreover, it has been shown that sumatriptan can inhibit trigeminal activation without its vasoconstrictive effects [26]. It has been postulated that sumatriptan exerts its antinociceptive effect by disrupting communication between peripheral and central trigeminovascular neurons [14]. In the current study, we showed that pre-treatment with sumatriptan was more effective in attenuating PACAP38induced headache. Ketorolac may exert its effect by suppression of central sensitization by directly silencing the peripheral and central trigeminovascular neurons [29]. Ketorolac treatment was more effective in terminating headache and allodynia in migraine patients who had established central sensitization [29]. Interestingly, we found that pre-treatment with ketorolac failed to prevent PACAP38-induced headache, though as mentioned a trend was observed. We suggest that the antinociceptive effect of ketorolac may partly depend on a prior activation of pro-inflammatory prostanoids.

PACAP38 degranulates dural mast cells and this mechanism may mediate PACAP38-induced prolonged arterial dilation [12]. Recent studies reported that PACAP38 caused degranulation of mast cells and histamine release via a specific receptor Mrgprb2 [22] which leads to prolonged activation of the trigeminal pain pathway [31]. In animals, sumatriptan inhibits mast cell degranulation [31], potently blocks neurogenic plasma protein extravasation from dural blood vessels [17] and prevents release of neuropeptides from perivascular neurons $[16,17]$. As a COX inhibitor ketorolac exerts its analgesic and anti-inflammatory effects via depression of prostanoid biosynthesis [43]. Prostaglandins and their receptors are widely distributed in the extraintracerebral arteries, trigeminal ganglion and trigeminal nucleus caudalis [43]. In addition to histamine dural mast cells can release various inflammatory mediators including prostaglandin $I_{2}$ that can activate and sensitize meningeal sensory afferents [51].

Taken together, the current study revealed that sumatriptan pre-treatment was more effective in preventing PACAP38-induced headache. This suggests that sumatriptan may have time dependent preventative properties which require further investigation.

\section{Conclusion}

The major finding of the present study was that no difference reported in PACAP38-induced headache after pre-treatment with sumatriptan or ketorolac. We found that post-treatment with ketorolac was more effective in attenuating PACAP38-induced headache compared to sumatriptan. Ketorolac exerted its effect without affecting PACAP38-induced arterial dilation, whereas sumatriptan post-treatment attenuated PACAP38-induced dilation of MMA and STA. Explorative analysis showed that pretreatment with sumatriptan attenuated PACAP38-induced headache without affecting PACAP38-induced arterial dilation.

\footnotetext{
Abbreviations

AUC: Under the curve; CAMP: Cyclic adenosine monophosphate; CGMP: Cyclic guanosine monophosphate:; CGRP: Calcitonin gene-related peptide; Cl: Confidence interval; COX: Cyclooxygenase; GTN: Glycerol trinitrate; HR: Heart rate; MAP: Mean arterial blood pressure; MCA: Middle cerebral artery; MMA: Middle meningeal artery; MRA: Magnetic resonance angiography; Mrgprb2: Mas-related G-protein-coupled receptors-b2; NO: Nitric oxide; PACAP38: Pituitary adenylate cyclase-activating polypeptide38; STA: Superficial temporal artery
}

Acknowledgements

The authors thank all participating healthy volunteers to the study. 


\section{Authors' contributions}

HG: study concept and design, acquisition, analysis and interpretation of data, and drafting and revision of manuscript. MAA: acquisition, analysis and interpretation of data, and drafting and revision of manuscript. NA: study concept and design, critical revision of manuscript for intellectual content. MMR and MMC: acquisition and analysis of data. HBWL and FMA: study concept and design, critical revision of manuscript for intellectual content. MA: study concept and design, interpretation of data, critical revision of manuscript, and study initiation and supervision. All authors read and approved the final manuscript.

\section{Funding}

The authors disclosed receipt of the following financial support for the research, authorship, and/or publication of this article: The study was supported by grants from Lundbeck Foundation (R155-2014-171).

\section{Availability of data and materials}

Anonymized data can be shared, until one year after publication, upon request to the corresponding author from qualified investigators for purposes of replicating procedures and results.

\section{Ethics approval and consent to participate}

All participants provided written consent to participate after receiving written and oral information in accordance with the Declaration of Helsinki of 1964, with subsequent revisions. The study was approved by the Ethics Committee of the Capital Region of Denmark ( $\mathrm{H}-18008313)$. The study was registered retrospectively at ClinicalTrials.gov (NCT03585894).

\section{Consent for publication}

Not applicable.

\section{Competing interests}

The authors declared the following potential conflicts of interest with respect to the research, authorship, and/or publication of this article: Messoud Ashina is a consultant, speaker or scientific advisor for Allergan, Amgen, Alder, ATI, Eli Lilly, Novartis, and Teva, primary investigator for Alder, Amgen, ElectroCore, Novartis and Teva trials. MA has no ownership interest and does not own stocks of any pharmaceutical company. MA serves as associated editor of Cephalalgia, Headache, and co-editor of the Journal of Headache and Pain. MA is the President of the International Headache Society. Mohammad Al-Mahdi Al-Karagholi has acted as an invited speaker for Novartis and received travel grant from ElectroCore, Hashmat Ghanizada, Nanna Arngrim, Faisal Mohammad Amin, Mette Mørch-Rasmussen, Matias Metcalf-Clausen and Henrik Bo Wiberg Larsson declare they have no conflicts of interest.

\section{Author details}

'Danish Headache Center and Department of Neurology, Rigshospitalet Glostrup, Faculty of Health and Medical Sciences, University of Copenhagen, Valdemar Hansens Vej 5, DK-2600 Glostrup, Denmark. ${ }^{2}$ Functional Imaging Unit, Department of Clinical Physiology, Nuclear Medicine and PET, Rigshospitalet, Faculty of Health and Medical Sciences, University of Copenhagen, Copenhagen, Denmark.

Received: 4 January 2020 Accepted: 12 February 2020

Published online: 24 February 2020

\section{References}

1. Akerman S, Goadsby PJ (2015) Neuronal PAC1 receptors mediate delayed activation and sensitization of trigeminocervical neurons: Relevance to migraine. Sci Transl Med 7:308ra157. https://doi.org/10.1126/scitransImed. aaa7557

2. Al-Karagholi MA-M, Ghanizada H, Hansen JM, Skovgaard LT, Olesen J, Larsson HBW, Ashina M (2019) Levcromakalim, an adenosine triphosphatesensitive Potassium Channel opener, dilates Extracerebral but not arteries, cerebral. Headache:1468-1480

3. Amin F, Lundholm E, Hougaard A, Arngrim N, Wiinberg L, de Koning PJ, Larsson HB, Ashina M (2014) Measurement precision and biological variation of cranial arteries using automated analysis of $3 \mathrm{~T}$ magnetic resonance angiography. J Headache Pain 15:25. https://doi.org/10.1186/1129-2377-15-25
4. Amin FM, Hougaard A, Schytz HW, Asghar MS, Lundholm E, Parvaiz Al, De Koning PJH, Andersen MR, Larsson HBW, Fahrenkrug J, Olesen J, Ashina M (2014) Investigation of the pathophysiological mechanisms of migraine attacks induced by pituitary adenylate cyclase-activating polypeptide-38. Brain 137:779-794

5. Amin FM, Asghar MS, Guo S, Hougaard A, Hansen AE, Schytz HW, van der Geest RJ, de Koning PJ, Larsson HB, Olesen J, Ashina M (2012) Headache and prolonged dilatation of the middle meningeal artery by PACAP38 in healthy volunteers. Cephalalgia 32:140-149. https://doi.org/10.1177/ 0333102411431333

6. Amin FM, Asghar MS, Ravneberg JW, De Koning PJ, Larsson HB, Olesen J, Ashina M (2013) The effect of sumatriptan on cephalic arteries: a 3T MRangiography study in healthy volunteers. Cephalalgia 33:1009-1016

7. Anzai M, Suzuki Y, Takayasu M, Kajita Y, Mori Y, Seki Y, Saito K, Shibuya M (1995) Vasorelaxant effect of PACAP-27 on canine cerebral arteries and rat intracerebral arterioles. Eur J Pharmacol 285:173-179

8. Arngrim N, Schytz HW, Britze J, Amin FM, Vestergaards MB, Hougaard A, Wolfram F, de Koning PJH, Olsen KS, Secher NH, Larsson HBW, Olesen J, Ashina M (2015) Migraine induced by hypoxia: a MRI spectrocopy and angiography study. Brain:723-737

9. Asghar MS, Becerra L, Larsson HBW, Borsook D. Calcitonin Gene-Related Peptide Modulates Heat Nociception in the Human Brain - An fMRI Study in Healthy Volunteers 2016:1-20

10. Asghar MS, Hansen AE, Kapijimpanga T, Van Der Geest RJ, Van Der Koning P, Larsson HBW, Olesen J, Ashina M (2010) Dilation by CGRP of middle meningeal artery and reversal by sumatriptan in normal volunteers. Neurology 75:1520-1526

11. Asghar MS, Hansen AE, Amin FM, van der Geest RJ, Van Der Koning P, Larsson HBW, Olesen J, Ashina M (2011) Evidence for a vascular factor in migraine. Ann Neurol 69:635-645

12. Baun M, Pedersen MHF, Olesen J, Jansen-Olesen I (2012) Dural mast cell degranulation is a putative mechanism for headache induced by PACAP-38. Cephalalgia 32:337-345. https://doi.org/10.1177/0333102412439354

13. Birk S, Sitarz JT, Petersen KA, Oturai PS, Kruuse C, Fahrenkrug J, Olesen J (2007) The effect of intravenous PACAP38 on cerebral hemodynamics in healthy volunteers. Regul Pept 140:185-191

14. Burstein R, Jakubowski M (2004) Analgesic Triptan action in an animal model of intracranial pain: a race against the development of central sensitization. Ann Neurol 55:27-36

15. Burstein $R$, Jakubowski $M$, Levy $D$ (2005) Anti-migraine action of triptans is preceded by transient aggravation of headache caused by activation of meningeal nociceptors. Pain 115:21-28

16. Buzzi MG, Dimitriadou V, Theoharides TC, Moskowitz MA (1992) 5 Hydroxytryptamine receptor agonists for the abortive treatment of vascular headaches block mast cell, endothelial and platelet activation within the rat dura mater after trigeminal stimulation. Brain Res 583:137-149. https://doi. org/10.1016/S0006-8993(10)80017-4

17. Buzzi MG, Moskowitz MA (1990) The antimigraine drug, sumatriptan (GR43175), selectively blocks neurogenic plasma extravasation from blood vessels in dura mater. Br J Pharmacol 99:202-206

18. Doenicke A, Brand J, Perrin VL (1988) Possible benefit of GR43175, a novel 5-HT1-like receptor agonist, for the acute treatment of severe migraine. Lancet (London, England) 1:1309-1311

19. Falkenberg K, Olesen J (2018) Pre-treatment with sumatriptan for cilostazol induced headache in healthy volunteers. J Headache Pain 19(1):71. https:// doi.org/10.1186/s10194-018-0890-y

20. Ghanizada H, Al-Mahdi Al-Karagholi M, Arngrim N, Ghanizada M, Wiberg Larsson HB, Amin FM, Ashina M (2019) Effect of pituitary adenylate cyclase-activating polypeptide-27 on cerebral hemodynamics in healthy volunteers: a 3T MRI study. Peptides 121:170134. https://doi.org/10.1016/j.peptides.2019.170134

21. Ghanizada H, Al-Mahdi Al-Karagholi M, Arngrim N, Olesen J, Ashina M. PACAP27 induces migraine-like attacks in migraine patients. Cephalalgia 2019;0:33310241986450. doi:https://doi.org/10.1177/0333102419864507

22. Green DP, Limjunyawong N, Gour N, Pundir P, Dong X (2019) A Mast-CellSpecific Receptor Mediates Neurogenic Inflammation and Pain. Neuron 101: 412-420.e3. https://doi.org/10.1016/j.neuron.2019.01.012

23. Hansen EK, Guo S, Ashina M, Olesen J (2016) Toward a pragmatic migraine model for drug testing: 1. Cilostazol in healthy volunteers. Cephalalgia 36:172-178

24. Hansen EK, Olesen J (2017) Towards a pragmatic human migraine model for drug testing: 2. Isosorbide-5-mononitrate in healthy individuals. Cephalalgia 37:11-19 
25. Héron A, Dubayle D (2013) A focus on mast cells and pain. J Neuroimmunol 264:1-7. https://doi.org/10.1016/j.jneuroim.2013.09.018

26. Hoskin KL, Kaube H, Goadsby PJ (1996) Sumatriptan can inhibit trigeminal afferents by an exclusively neural mechanism. Brain 119:1419-1428

27. Humphrey PP, Feniuk W, Marriott AS, Tanner RJ, Jackson MR, Tucker ML (1991) Preclinical studies on the anti-migraine drug, sumatriptan. Eur Neurol 31:282-290

28. Iversen H, Olesen J (1996) Headache induced by a nitric oxide donor (nitroglycerin) responds to Sumatriptan. A human model for development of migraine drugs. Cephalalgia 16:412-418. https://doi.org/10.1046/j.14682982.1996.1606412.x

29. Jakubowski M, Levy D, Goor-Aryeh I, Collins B, Bajwa Z, Burstein R (2005) Terminating migraine with allodynia and ongoing central sensitization using parenteral administration of COX1/COX2 inhibitors. Headache 45:850861

30. Jansen-Olesen I, Hougaard Pedersen S. PACAP and its receptors in cranial arteries and mast cells. J Headache Pain 2018;19

31. Levy D, Burstein R, Kainz V, Jakubowski M, Strassman AM (2007) Mast cell degranulation activates a pain pathway underlying migraine headache. Pain 130:166-176

32. Levy D, Jakubowski M, Burstein R (2004) Disruption of communication between peripheral and central trigeminovascular neurons mediates the antimigraine action of $5 \mathrm{HT} 1 \mathrm{~B} / 1 \mathrm{D}$ receptor agonists. Proc Natl Acad Sci U S A 101:4274-4279

33. Miyata A, Arimura A, Dahl RR, Minamino N, Uehara A, Jiang L, Culler MD, Coy DH (1989) Isolation of a novel 38 residue-hypothalamic polypeptide which stimulates adenylate cyclase in pituitary cells. Biochem Biophys Res Commun 164:567-574

34. Miyata A, Jiang L, Dahl RD, Kitada C, Kubo K, Fujino M, Minamino N, Arimura A (1990) Isolation of a neuropeptide corresponding to the N-terminal 27 residues of the pituitary adenylate cyclase activating polypeptide with 38 residues (PACAP38). Biochem Biophys Res Commun 170:643-648

35. Moller K, Zhang YZ, Hakanson R, Luts A, Sjolund B, Uddman R, Sundler F (1993) Pituitary adenylate cyclase activating peptide is a sensory neuropeptide: immunocytochemical and immunochemical evidence. Neuroscience 57:725-732

36. Moskowitz MA (1993) Neurogenic inflammation in the pathophysiology and treatment of migraine. Neurology 43:S16-\$20

37. Pardutz A, Schoenen J (2010) NSAIDs in the acute treatment of migraine: a review of clinical and experimental data. Pharmaceuticals 3:1966-1987

38. Pedersen SH, la Cour SH, Calloe K, Hauser F, Olesen J, Klaerke DA, JansenOlesen I (2019) PACAP-38 and PACAP (6-38) Degranulate rat meningeal mast cells via the orphan MrgB3-receptor. Front Cell Neurosci 13:1-11. https://doi.org/10.3389/fncel.2019.00114

39. Peroutka SJ, McCarthy BG (1989) Sumatriptan (GR 43175) interacts selectively with 5-HT1B and 5-HT1D binding sites. Eur J Pharmacol 163:133-136

40. Reuter U (2001) Delayed inflammation in rat meninges: implications for migraine pathophysiology. Brain 124:2490-2502

41. Rice ASC, Lloyd J, Bullingham RES, O'Sullivan G (1993) Ketorolac penetration into the cerebrospinal fluid of humans. J Clin Anesth 5:459-462

42. Schytz HW, Birk S, Wienecke T, Kruuse C, Olesen J, Ashina M (2009) PACAP38 induces migraine-like attacks in patients with migraine without aura. Brain 132:16-25

43. Silberstein SD, Stirpe JC (2014) COX inhibitors for the treatment of migraine. Expert Opin Pharmacother 15:1863-1874. https://doi.org/10.1517/14656566. 2014.937704

44. Sinha V, Kumar R, Singh G (2009) Ketorolac tromethamine formulations: an overview. Expert Opin Drug Deliv 6:961-975. https://doi.org/10.1517/ 17425240903116006

45. Theoharides TC, Donelan J, Kandere-Grzybowska K, Konstantinidou A (2005) The role of mast cells in migraine pathophysiology. Brain Res Rev 49:65-76

46. Tvedskov JF, Iversen HK, Olesen J, Tfelt-Hansen P (2010) Nitroglycerin provocation in normal subjects is not a useful human migraine model? Cephalalgia 30:928-932

47. Valledor AF, Comalada M, Santamaría-Babi LF, Lloberas J, Celada A (2010) Macrophage Proinflammatory activation and deactivation. Quest Balance Adv Immunol 108:1-20

48. Vane JR, Botting RM (1998) Mechanism of action of nonsteroidal antiinflammatory drugs. Am J Med 104:2S-8S discussion 21S-22S

49. Vaudry D, Falluel-morel A, Bourgault S, Basille M, Burel D, Wurtz O, Fournier A, Chow BKC, Hashimoto H, Galas L, Vaudry H, National I, Sante D (2009)
Pituitary Adenylate Cyclase-activating polypeptide and its receptors : 20 years after the discovery. Pept Res 61:283-357. https://doi.org/10.1124/pr. 109.001370.283

50. Zhang XC, Kainz V, Burstein R, Levy D (2011) Tumor necrosis factor-a induces sensitization of meningeal nociceptors mediated via local COX and p38 MAP kinase actions. Pain 152:140-149. https://doi.org/10.1016/j.pain. 2010.10.002

51. Zhang XC, Strassman AM, Burstein R, Levy D (2007) Sensitization and activation of intracranial meningeal nociceptors by mast cell mediators. J Pharmacol Exp Ther 322:806-812

52. Zhang YZ, Sjo B, Moller K, Håkanson R, Sundler F (1993) Pituitary adenylate cyclase activating peptide produces a marked and long-lasting depression of a C-fibre-evoked flexion reflex. Neuroscience 57:733-737

53. Ødum L, Petersen LJ, Skov PS, Ebskov LB (1998) Pituitary adenylate cyclase activating polypeptide (PACAP) is localized in human dermal neurons and causes histamine release from skin mast cells. Inflamm Res 47:488-492

\section{Publisher's Note}

Springer Nature remains neutral with regard to jurisdictional claims in published maps and institutional affiliations.
Ready to submit your research? Choose BMC and benefit from:

- fast, convenient online submission

- thorough peer review by experienced researchers in your field

- rapid publication on acceptance

- support for research data, including large and complex data types

- gold Open Access which fosters wider collaboration and increased citations

- maximum visibility for your research: over $100 \mathrm{M}$ website views per year

At BMC, research is always in progress.

Learn more biomedcentral.com/submissions 